RACAR : Revue d'art canadienne

Canadian Art Review

RACAR

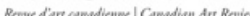

\title{
Gérard Morisset (1898-1970) et le portrait au Québec : reflets d'un idéal canadien-français
}

\section{Mickaël Bouffard-Veilleux}

Volume 34, numéro 2, 2009

URI : https://id.erudit.org/iderudit/1069485ar

DOI : https://doi.org/10.7202/1069485ar

Aller au sommaire du numéro

\section{Éditeur(s)}

UAAC-AAUC (University Art Association of Canada | Association d'art des universités du Canada)

\section{ISSN}

0315-9906 (imprimé)

1918-4778 (numérique)

Découvrir la revue

\section{Citer cet article}

Bouffard-Veilleux, M. (2009). Gérard Morisset (1898-1970) et le portrait au Québec : reflets d'un idéal canadien-français. RACAR : Revue d'art canadienne/ Canadian Art Review, 34(2), 5-19. https://doi.org/10.7202/1069485ar
Résumé de l'article

Gérard Morisset (1898-1970) can be numbered among the most important founders of Québec's art history. This article highlights the ideology underlying Morisset's analyses and descriptions of French Canadian portraiture. Through a variety of texts published between 1936 and 1960, Morisset constructs a history of portraiture in the province, systematically using that history to praise a French Canadian national character. He claims to be able to read honesty, industry, simplicity and a "smiling bonhomie," not only in the facial features of French Canadian sitters but also in the brushstrokes of Québec's francophone portraitists.
Tous droits réservés @ UAAC-AAUC (University Art Association of Canada | Association d'art des universités du Canada), 2009
Ce document est protégé par la loi sur le droit d'auteur. L'utilisation des services d’Érudit (y compris la reproduction) est assujettie à sa politique d'utilisation que vous pouvez consulter en ligne.

https://apropos.erudit.org/fr/usagers/politique-dutilisation/ 


\title{
Gérard Morisset (1898-1970) et le portrait au Québec : reflets d'un idéal canadien-français
}

\author{
MickaËl Bouffard-Veilleeux, Université de Montréal
}

\begin{abstract}
Gérard Morisset (1898-1970) can be numbered among the most important founders of Québec's art history. This article highlights the ideology underlying Morisset's analyses and descriptions of French Canadian portraiture. Through a variety of texts published between 1936 and 1960, Morisset constructs a history of portraiture in the province, systematically using that history to praise a French Canadian national character. He claims to be able to read honesty, industry, simplicity and a "smiling bonhomie," not only in the facial features of French Canadian sitters but also in the brushstrokes of Québec's francophone portraitists.
\end{abstract}

\section{Introduction}

C'est que le portrait, quoi qu'on en pense parfois, est la sauvegarde naturelle de la peinture saine. Dans le portrait, point de tricherie-car le bourgcois ne badine pas sur la ressemblance; point de laisser-aller-car l'auguste portraituré exige que son peintre ait bien en mains sa monture de procédés, son entière collection de couleurs, toute l'éloquence de ses moyens techniques; point de copie-car tout commerce avec le déjà vu serait une atteinte grave à l'éminente pcrsonnalité de celui qui daigne poser devant le peintrel.

C es mots dithyrambiques à l'égard du portrait expriment la pensée de Gérard Morisset (1898-1970), qui peut être considéré aujourd'hui comme l'un des fondateurs de l'histoire de l'art du Québec ${ }^{2}$. Lc portrait occupe une place de premier choix dans son œuvre historique, non seulement parce que ce genre pictural fut l'un des plus considérables de l'art ancien québécois, mais aussi parce que, comme le suggère la citation précédente, le portrait aurait joué un rôle salvateur au Canada français.

Cette idée, il l'a déjà publiée et la republiera. Dans son livre Coup d'xil sur les arts en Nouvelle-France (1941), il avait même été jusqu'à dire: "Plus on a dit du mal du portrait, plus le portrait s'est avéré comme la sauvegarde naturelle de la peinture saine. [...] En sorte qu'on peut affirmer qu'en Nouvelle-France comme ailleurs le portrait a sauvé la peinture ${ }^{3}$. "Pour cet historien de l'art, le portrait, plus que tout autre genre, a forcé les artistes à se soumettre à une forme de réalisme, à humblement prêter attention aux détails du monde récl, contrairement à la peinture religieuse qui aurait plutôt encouragé la copie servile et le pastiche des grands maîtres. Considérant que son époque vivait un déclin social et culturel ${ }^{4}$ et que le portrait n'était plus assez présent pour ramener les artistes à produire un art sain 5 , il crut bon de solliciter le secours de la bourgeoisie par l'intermédiaire du journal Le Droit. Il exhorta celle-ci à commander des portraits pour que les artistes contemporains pussent, comme ceux d'autrefois, "développer leur talent ${ }^{6}$ ". Le réalisme est ici, comme nous le verrons, affaire d'éthique professionnelle et d'honnêteté, des vertus qui, dans l'esprit de Morisset, apparaissaient comme des traits traditionnels du peuple canadien-français.
Le thème du portrait lui permit donc de développer un idéal très valorisant de ses compatriotes. Dans un premier temps, en esquissant les biographies des portraitistes du Québec, Morisset en profitait pour vanter lcur vaillance au travail ainsi que leur honnêteté. Dans un deuxième temps, la description des portraits eux-mêmes donnait lieu à l'attribution de quelques traits de caractère récurrents que Morisset prétendait lire dans les traits faciaux des Canadiens français portraiturés : la simplicité, la bonne humeur, l'amabilité et la bonhomie souriante. En somme, que ce soit en parlant du portraitiste ou en livrant une description de son modèle, l'autcur peignait lui-même un portrait-littéraire, idyllique et idéologique-de la société traditionnelle canadienne-française. En analysant le propos historique porté par Morisset sur ce genre pictural, on se rend vite compte qu'un discours à teneur nationaliste s'y mêle indissociablement comme, dans le tissage, les fils de trame s'unissent aux fils de chaîne.

Avec l'intention de démêler les fils de ce tissu serré, nous débutcrons par une analyse approfondie du thème du portrait à travers tout l'œuvre écrit de Gérard Morisset 7 . Ce faisant, il nous faudra d'abord qualifier son approche du portrait et la distinguer de celle de ses prédécesseurs; ensuite, nous nous attarderons sur cet idéal du Canadien français honnête, travaillant, bienveillant et de bonne humeur qui intervient systématiquement lorsque Morisset aborde ce genre pictural. En deuxième partie, nous poursuivrons notre étude en nous efforçant de situer ce type de discours nationaliste non sculement dans le parcours personnel de l'auteur, mais aussi dans le cadre idćologique de son époque.

\section{Morisset et son discours sur le portrait}

\section{Approche du portrait}

Les premières publications marquant de l'intérêt pour ce genre pictural sont celles que diffuse la Numismatic and Antiquarian Society of Montreal. Il s'agit de catalogues d'expositions organisées en 1887, puis en 1892 , dont le contenu préfigurait la future collection du Château Ramezay ${ }^{8}$. On y retrouve toujours le même type d'informations: notes biographiques sur la personne portraiturée, identification de la technique (huile sur toile, pastel, aquarelle ...) et, plus rarement, tentative d'attribution à 


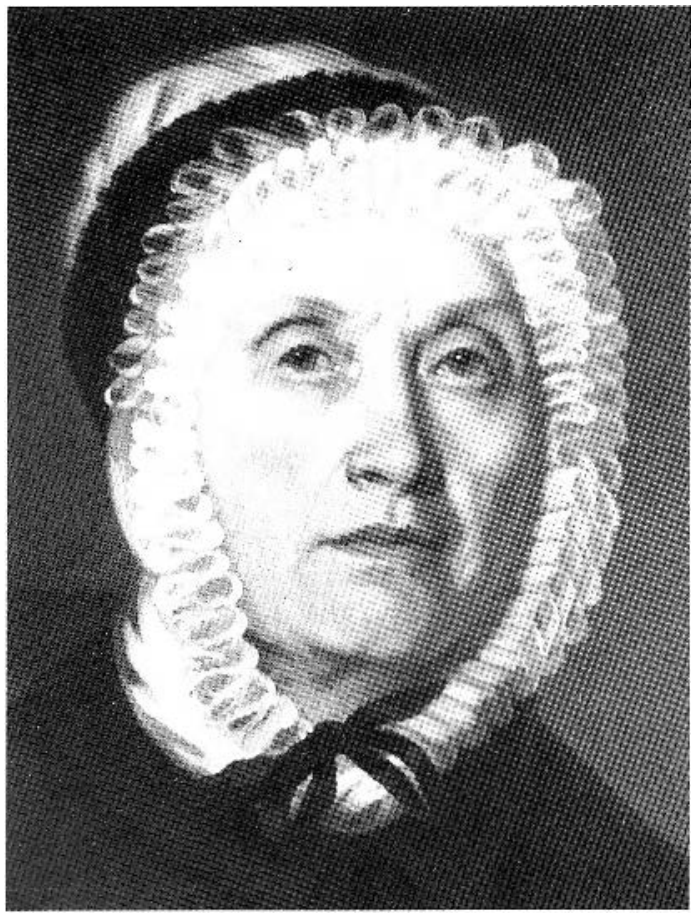

Figure 1. Théophile Hamel, Sophria Melvin Ploce (dćtail), 1851. hulle sur toile, $90 \times 71 \mathrm{~cm}$. Qućbec.. Musée Bon-Pastcur (crćdit pholographique Musée Bon-Pasteur)

un artiste. De toutc évidence, l'intérêt historique prédomine. Considćrant l'engonement qu'ont suscité les grandes figures de l'histoire durant la seconde moirić du XIXe siècle, ce type d'exposition servait un goût historiciste qui se plaisait à mettre des visages sur des noms célc̀bres. I e portrait n’esı donc pas traité différcmment des artefacts canadicns (cartes géographiques anciennes, pićces de monnaie, armes, objets amćrindicns, livres anciens ...) cxposés à ses côtés.

À la lumicre de ces maigres antécédents, on constate que Morissct est le premier à tenir un véritable discours sur l'art du portrait au Québec. Pour aborder celui-ci, il utilise ce que Maurice I emire a identifić commc sa technique descriptive, qui s'assortit presque toujours d'unc interprétation psychologiquc"). Voici un extrait de la description du portrait de madame Sophie Melvin-Place (fig. 1) : "Sophic Mclvin est toute vêtuc de noir : clle porte un bonnet fraisé blanc, nouć d'un ruban de satin noir. Ellc est loin d'être jolie. Mais sa figurc plaît par la placidité du regard, l'énergie de sa bouche ct du monton, l'ascétisme des yeux creux et des joucs amaigries. Ce sont les traits d'unc brave femme volontaire et généreuse ${ }^{10}$. "Même dans son roman Novembre 1775, on sent que l'historien de l'art n'est jamais bicn loin derrière l'écrivain. Dans l'extrait suivant, le personnage principal est en train d'obscrver le mobilier de la veuve Loubicr :
Au-dessus de la cheminćc, Ic portrait de François Loubier, en grande perruque ec en jabot de dentelle, évoque l'image du fonctionnaire ponctud et intraitable. Fn face, c'est le portrait de sa troisième femme à vingt-deux ans, Grand'mère con personne, fraîche et presque jolie; il y a un soupçon do tendresse dans la courbe du nez; le reste est d'une durcté de méal : les yeux d'un bleu acide, le front bas, le menton qui s'étale sous deux lèvres minces, le porr de tête, tour dans ce portrait est insupportablell.

Morisset ne fait pas sicnne n'importe quelle technique descriptive d'analyse psychologique; le fait de reconnaittre dans certains traits ccux d'une "brave femme volontaire et généreuse " ou dc déceler un " soupçon de tendresse dans la courbe du nez " trahit une approche physiognomoniste du portrait. La physiognomonie est cette science qui prétcendait pouvoir lire l'âme par les apparences du corps, interpréter unc personnalité aux moyens de signes extćricurs, comme les traits du visage ${ }^{\prime 2}$. Morisset va parfois fairc un usagc abusif de ce type de lecture, comme en témoignc le passage qui suit -il s'agit du portrait de l'abbé l ouisPhilippe Desjardins par Antoine Plamondon (fig. 2) :

Il est vu de face. Sur sil tête massive, une grande calortc noire. la figure bien mise en lumiere, le front haut et large, les paupières lourdes, les prunclles fixes, le nez long et recourbé, les joucs tombantes, les lèvres épaisses er malicieuses, les bouckes grisonnantes encadrant le visage, bref la physionomic cntière cmpreinte de bonhomie est exrraordinairement vivante er finc. On perçoir un peu d’égoüsme dauns la partic inféricure du visange, de la roucric dans l'assurance du regard, du désabusement dans l'expression 1.3.

Or, ce n'est pas toujours sur le ton de la méraphore que Morisset ćtablit des analogies cntre trait facial et trait de caractère. Sa description d'un portrait gravé de Samucl de Champlain en témoignc : "Sous l'habit, sorte de casaque dont les basques descendent jusquaux genoux, on devine la robustesse de son corps solidement planté sur de grosses jambes. Les traits du personnage sont forts, le front bombé, lc ncz énorme. Il n'y a aucune raison de croire que ce portrait n'cst pas fidèle, car tout $y$ reflète l'âme du fondateur de (Qućbcc ${ }^{1}$. " Il déclare l'effigie fidèle, puisclu'elle lui inspire les traits de caractìre que les biographes du fondateur de Québec lui atribuent courammont. Dès lors que l'article s'efforce de départager les reprćscntations imaginaires de Champlain de celles qui sont authentiques, on s'étonne que l'analyse physio-gnomoniste puisse servir à assurcr la véracité de ce portrait.

\section{Idéal du Canadien français}

Dans le texte du catalogue de l'Exposition rétrospective de l'art au Canada français (1952), Gérard Morisset passe de l'analyse physiognomonicue de l'individu à celle de la nation : 
Cependant rcllc qu'elle est, cette peinture reflictc avec beaucoup de fiććlitć les caractères du petit peuple quiclle représente. Pcuplc d'armateurs, d'artisans et dc boutiquiers; peuple de traitants en fourrures, de coureurs de bois et d'aventuriers, dc hobereaux ruinés mais férus de noblesse, et de paysans à l'aise; peuple profondément rcligicux et attaché à ses pastcurs; enfin peuple actif, réaliste ct dc bonne humeur, tel on lc voit dans les portraits ici rassemblćs : peu soigneux de sa personne mais fort attentif devant le peintre, gravc ou souriant, mais toujours sympathique, bien vivant. À défaut d'élégance dans le dessin ct de finesse dans le coloris, le portrait canadien possède une qualité qui lui est propre, la vivacité dans le réalisme. Et sous la lourdcur de la touche-qu'elle soit de Cardenat, de Louis de Heer ou de François Baillairgć-, on sent l'application du peintre à faire urai et à rendre sur la toile, avec le moins de complications possible, la vic qui est devant ses yeux sous les traits d'un prélat, d'un administrateur sérieux, d'un bourgeois épanoui, d'unc jcune femme intimidée ou d'un prêtre qui pose simplementis.

Pour Morisset, la peinture canadienne-française-et plus particulièrement le portrait-reflète le caractère de ce " petit peuple " qu'il décrit comme " actif, réaliste et dc bonne humeur, $[\ldots]$ grave ou souriant, mais toujours sympathique ${ }^{16}$ ". L'historien de l'arc a soutenu maintes fois l'idée que la peinture pouvait servir à déchiffrer l'âme d'une nationn ${ }^{17}$. Il étend même cette proprićté au mobilier, qui portcrait - supposet-il- "le sourirc de la race ${ }^{18}$ ". Le portrait canadicn possède aussi comme qualité en propre la "vivacité dans le réalisme". Cette caractéristique devait à elle seule racheter la piètre qualité plastique des portraits choisis pour l'Exposition rétrospective de l'art au Canada français. Cette "application du peintre à faire vrai "contient plusieurs enjeux éthiques qui relèvent du caractère moral de la nation, un point sur lequel nous reviendrons ultérieurement.

L'idée que le portrait reflète l'âme du peuple sc retrouve intégralement appliquće à la production (supposéc) dc François Baillairgé comme portraitiste (fig. 3) :

Tout leur intérêt artistique réside dans une sorte de réalisme paysan, naïvement sincère, plein de familiaricé ct de bonhomie; réalisme d'artisan jovial et sans façon, tout d'unc pièce, mais avec des finesses à peine murmurćes, des sourires narquois, des sous-cntendus spirituels, de bonncs grosses histoires débitćcs à demi-mots, un sentiment humain d'une chaleurcusc cordialité; réalisme de la vision ct rćalisme du pinceau, avec juste ce qu'il faur d'artificielle cr de tenue pour ne pas tomber dans le vulgaire, avec juste cc quil faut de vivacité, de naturalisme et de couleur locale pour se distinguer d'une certainc pcinture fade er ennuyeuse.

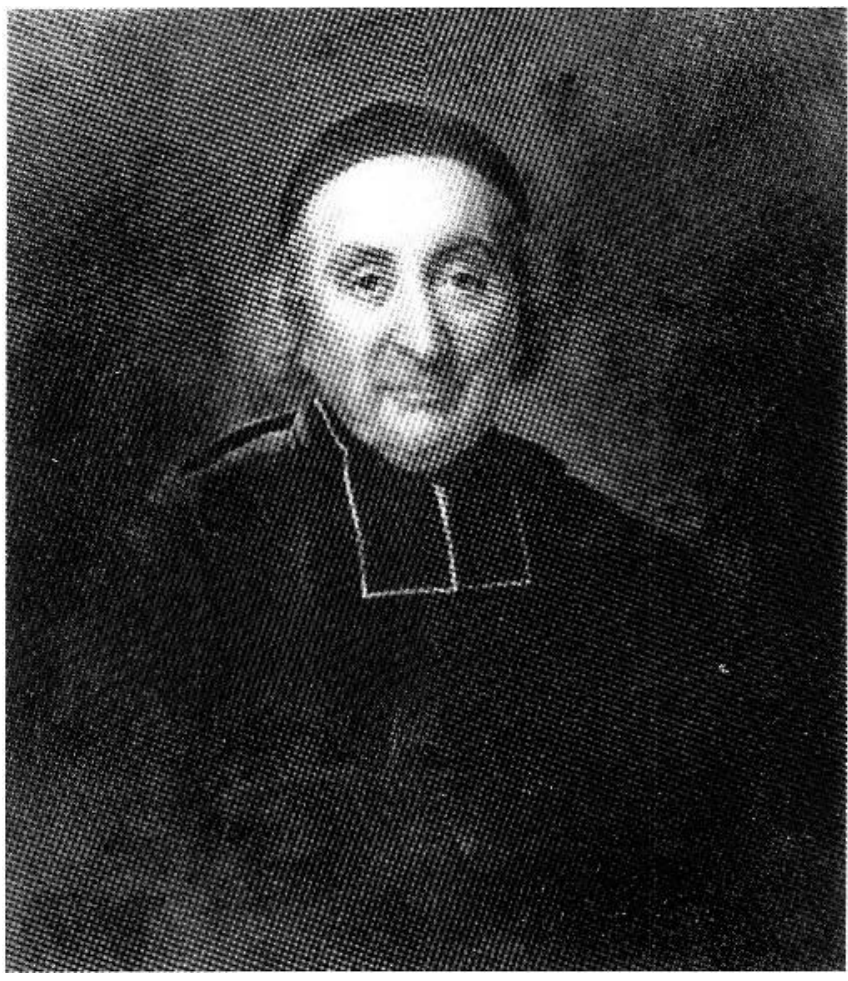

Figure 2. Antoıne Plamondon (d'après Paulin Guérin). L'ıbbé Louis-Philippe Desjardins, 1828-30. huile sur toile, $72.3 \times 60.3 \mathrm{~cm}$. Musée des Augustines de l'Hôtel-Dieu de Québec (crédit photographique : Musée des Augustınes).

Le plus intéressant de ces portraits est assurćment celui de François Ranuoyzé; il a été peint vers 1790. Le grand orfêvre y paraît affablo, accueillant, de belle humcur; même au repos, ses traits restent souriants; son expression est celle d'un homme qui manic fincment et goûte la plaisanteric; de sa physionomie délurćc sc dégage une indolence conscicnte enveloppée d'un sourire; le fond de son caractèrc, c'est le goût du fignolagc, la fantaisie de son imagination, le caprice. L'ćtude de l'cuvre de Ranvoyzé, surtout son style décorarif, confirme les traits de caractère de ce portrait; er l'on restc ćınné que le peintre ait pu, d'unc main lourde er de médiocres coulcurs, rendre d'une manic̀re si juste er si subrile la physionomie morale d'un quant-à-soi habituellement réservél').

Ce long extrait déploie tout un champ lexical qui tourne autour de la bonhomic : familiarité, jovial, sourires, chaleureuse cordialité, affablc, accueillant, belle humeur, souriants, plaisanterie. Ce sont autant de termes qui contribuent à construirc la " physionomie morale " que Morisset applique autant au Canadien français qui portraiture qu'à celui qui est portraituré. Dans le même extrait, l'usage de l'expression "réalisme paysan naïvement 


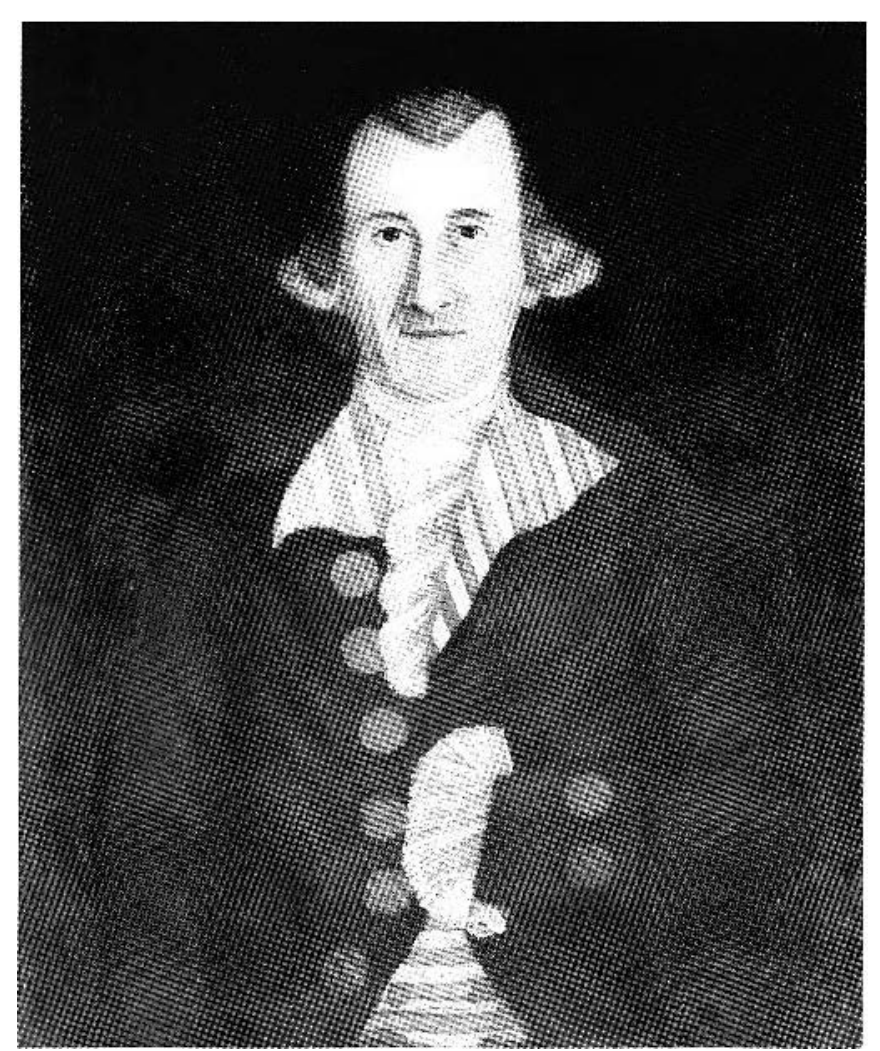

Figure 3. Louis-Chrétien de Heer (attrıbué à), Fronçoıs Ranvoyzé, vers 1790. huile sur toile, $66.4 \times 54 \mathrm{~cm}$. Musée natıonal des beaux-arts du Québec. inv. 98.19 (crédit photographique : MNNAQ. Patrick Altman). Morısset l'avaıt attribué à François Baillairgé.

sincère " introduit un second leitmotiv. L'idée d'associer réalisme et sincérité peut paraître étrange, mais elle s'affirme commc une constante dans la production écrite de Morisset. La citation précédente contienc donc les deux grands pôles de valorisation du peuple canadien-français qui se glissent systématiquement à travers le discours historique de Gérard Morisset sur le portrait : l'honnêteté et la bonhomie. Ellc illustre également les deux façons dont il s'y prend pour accomplir cette valorisation : tantôt en décrivant le travail essentiellemcnt probe du portraitiste, tantôt en faisant l'ekphrasis de ceux qui posent.

\section{Honnêteté et réalisme}

Alors qu'il décrit le travail de Napoléon Bourassa (dont il hait viscéralement la pcinture d'histoire), Morisset se fait élogieux lorsqu'il en vient à parler de ses portraits : "Dans le portrait, un homme loyal et méticuleux comme Napoléon Bourassa nc peut produire que de la peinturc honnête, sans panache, distinguée. [...] Au Collège de Saint-Hyacinthe, une quinzaine de portraits, marquent, beaucoup mieux que le reste de son œuvre, le talent probe, précis, limité, de Bourassa ${ }^{20}$. "Ici, ce sont les mots "loyal ", " méticuleux ", " honnête " et " probe " qui servent à qualifier Bourassa et sa peinture. La même appréciation sera faite du peintre Antoine Plamondon ${ }^{21}$. La valorisation de l'honnêteté dans l'art du portrait est quasi systématique. Mais en quoi consiste exactement l'honnêteté quand on portraiture?

La réponse se trouve dans la citation qui ouvre cette étude. On en déduit qu'elle se caractérise d'abord par l'absencc de tricherie quant à la ressemblance, c'est-à-dire par la volonté de se soumettre au réel et de l'observer. Elle consiste aussi à refuser le laisser-aller, donc à s'appliquer avec minutie et à ne pas lésiner paresseusement sur les moyens. Enfin, elle revient à éviter la copie ou le plagiat que Morisset associc généralement à la peinture d'histoire religieuse.

De toutes ces caractéristiques, c'est surtout celle du réalisme de l'effigie qui prédomine dans la pensée de Morisset. En parlant du portrait canadien aux XVIIle et XVIIIe siècles, il dira d'ailleurs que ce " sont des effigies peintes d'une main à la fois méticuleuse ct lourde, quasi photographiques à force de conscience et d'autant plus vraies que les artistes, en les brossant laborieusement, n'ont qu'une préoccupation : parvenir à la ressemblance parfaite du modèle 22 ". C'cst donc à force de conscience (professionnelle ?) que le peintre s'applique à rendre le réalisme de la figure. Il ne s'agit donc pas d'un réalisme à la manière de Van Eyck ni de Courbct. Par rapport à ces deux types de réalisme, on ne peut pas dire que le portrait canadien soit " réaliste". Ce qualificatif fait plus référence aux bonnes intentions de l'artiste dans l'observation du modèle qu'au résultat final sur la toilc.

Cette valorisation du portraitiste et du portrait canadienfrançais à travers les qualitćs de probitć ou d'honnêtcté s'articule autour d'une expression récurrente dans l'œuvre de Morisset : faire vrai. Le meilleur exemple est sans conteste tiré de son commentaire sur Jean-Baptiste Roy-Audy : "Faire vrai, c'est toute son ambition; c'est la fin ultime de son art; c'est une affaire d'honnêteté professionnelle et de respect du client. Quand celui-ci veut se payer un portrait peint de quinze louis, il faut qu'il en ait pour son argent; $[\ldots]^{23}$. " On décèle ici la dimension éthique qu'implique le réalisme : l'éthique professionnelle attendue chez un bon artisan. Contrairement à ce qu'on pourrait supposer, l'expression faire vrai nc décrit pas la singularité de l'art de Roy-Audy. Morisset l'utilise pour ainsi dire à propos de tous les portraitistes, que ce soit Antoine Plamondon : "Il s'agit de camper ses personnages à l'avantage de leur physique, de saisir la ressemblance des visages et des accessoires; il s'agit de faire vrai24. "; ou bien Napoléon Bourassa : "Dans ce genre, point de théorie ni d'idée; il s'agit de faire vrai, [...]. La rćalité ramène Bourassa à une saine compréhension de la peinture ${ }^{25}$. "; ou encore Théophile Hamel : "Et au lieu de vouloir peindre une belle image, pimpante et pommadée, il a cherché à rendre ce qu'il voyait, avec le seul souci de faire vrai ${ }^{26}$. "Louis Dulongpré, un Français immigré sous le régime anglais, peut lui aussi 
prétendre à cettc conception idéale, pour autant qu'il adhère aux traditions canadiennes déjà en place : "De là le caractère photographique de son couvre, une certaine sécheressc due aussi bien à son modelé laborieux qu'à sa préoccupation constante de faire vrai. Ainsi le peintre reste-t-il dans la tradition canadienne et rejoint-il par sa méticuleuse application la manière froide des portraitistes de l'École de Saint-Joachim ${ }^{27}$."

Morisset expose ici cette même idée voulant que la " vivacité dans le réalisme " soit le propre de l'art du portrait canadien ${ }^{28}$. Il ne faut toutefois pas se laisser mener si facilement par l'auteur : plus qu'il n'a isolé le propre du portrait réalisé au Québec, Morisset a repris l'un des plus célèbres topö̈ associés au discours sur le portrait. Comme l'a montré Édouard Pommier, "la minutie dans la conformité au modèle [comme] critère de réussite du portrait " est une notion déjà bien courante dans les théories artistiques du XVIe siècle et prend sa source dans le débat antique sur l'imitation de la nature ${ }^{29}$. L'originalité de Morisset, semble-t-il, c'est son insistance à faire du réalisme d'un portrait une affairc d'honnêteté.

\section{Bienvellance et bonhomie : autour du sourire}

Morisset tente de tirer un autre trait moral de sa lecture du portrait peint au Canada français. On en retrouve l'indice dans un passage cité plus tôt où il décrit ce " peuple actif, réaliste et de bonne humeur [...], grave ou souriant, mais toujours sympathique ${ }^{30}$ ". Tantôt la bonhomie, tantôt la bienveillance sont évoquées, mais dans les deux cas, Morisset fait souvent appel à unc conception quasi mystique du sourire.

l a description du portrait de l'abbé F́douard Faucher, ancien curé de lotbinière, réalisé par 'Ihéophile Hamel est asse\%. typique des ekphraseis de notre auteur :

[...] force tranquille, de bonne humeur er volontiers narquoise; car dans certe tête ronde où le double menton répond à la courbe du crâne à demi dénudć, il y a la malicc plaisantc du regard et l'ourlet sarcastique de la lèvre inféricurc; $|. .$. la monumentalitć du personnage y est tempérée par une fine bonhomie, par un maintien majestucux qui n'exclut pas la fermeté ni la souplesse, par une attention constance de l'esprit qui domine la marière et l'ennoblit ${ }^{31}$.

Cette description joviale de l'abbé Faucher, à laquelle le modèle invite sans aucun doute, contient des éléments qu'elle partagc avec maintes autres descriptions. Pour le portrait du frère I ouis Bonami, qu'il attribue à François Baillairgć, Morisset ćcrit : "C' Cst un solide gaillard au teint coloré, à la mine réjouic, au sourire facile; [... $]^{32}$. "Les portraits de Louis Baribault et de sa femme sont dits "pleins de bonhomie bourgeoise ${ }^{33}$ ". Quant aux mćplats du visage de madame François Poulin de Courval, ils " accuscnt la douceur et la bienveillance de ce visage un peu fanć, et la suave fixité du regard; $[\ldots]^{34}$ ". Il serait inutile de

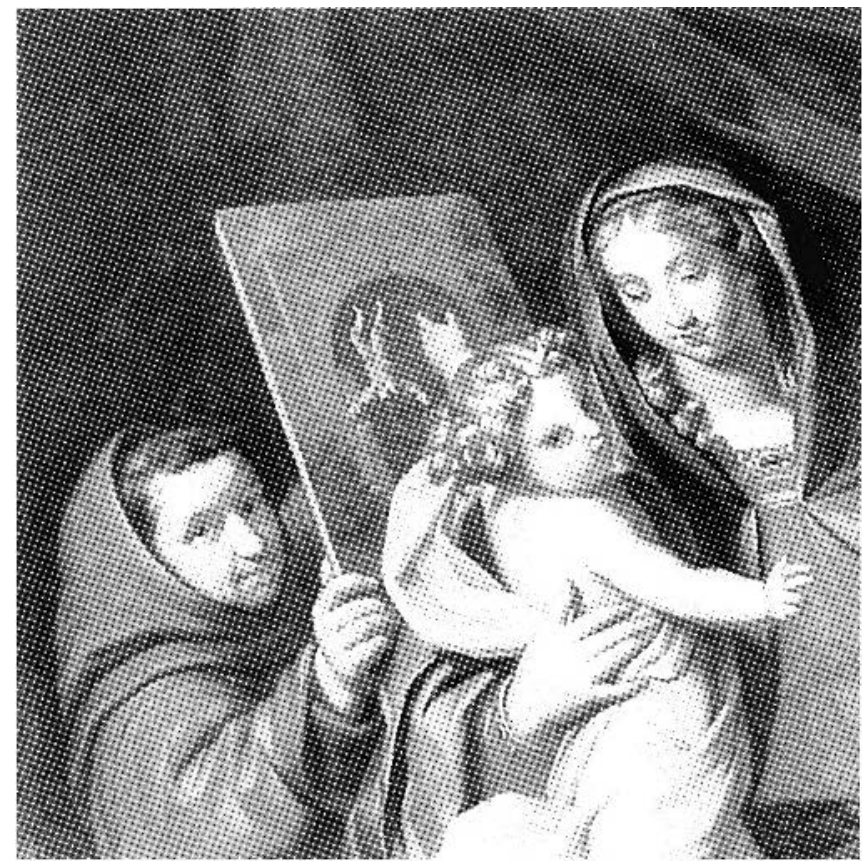

Figure 4. Claude François (dit frère Luc). Ex-voto ò Notre-Dame de Foy (détaii). milieu du XVIle sièclc, huilc sur toile. $2.35 \times 1.70 \mathrm{~m}$. Église de Ncuvillc-lcss-Loeuilly (crédıl pholographique : Alain Dulın).

multiplier les excmples d'unc redondance déjà bien évidente. Les mots " sourire " et "bonhomie " hantent l'écriture de Gérard Morisset. Ce visage du peuple canadien-français qu'il dépeint dans ses ouvrages d'histoirc de l'art nous est également servi dans son roman. Julien, le personnage principal, y croise le peintre François Baillairgé ct l'orfevre François Ranvoyzé en plcinc discussion près de la cathédrale de Québec : "Julien observe ce petit homme déjà ridé, à l'œil malicicux et au sourire narquois; c'est l'orfèvre. Il sc le figurait tout autre : grand, maigre, tiré à quatre épingles, le visage sćvère et le comportement un tantinct cmpesć; il a fait erreur; il reconnaît que l'artisan est plus sympathique tel qu'il est, avec son long nez fouinard, sa bonhomic ct son veston râpé35. "Comme on peut le constater, il ne décrit pas l'orfèvre différemment des personnages posant dans les portraits qu'il commente.

Ce qui est surprenant par contre, c'est de le voir décrire ces mêmes traits de caractère dans des effigies qui ne le suggèrent aucuncment. La description de l'autoportrait du frère l uc en est un bon excmple (fig. 4) :

On se rappelle l'Ex-voto de Neuville-lès-I oeuilly; il conrient le portrait même du Frère l.uc [...]. Il s'en dégage l'affirmation d'une personnalitć, mais laqucllc? Jc n'oscrais l'analyscr trop en profondeur de crainte d'y trouver, sous une écorce un peu rude, un bon bonhomme de moinc, simple, jovial, facć- 


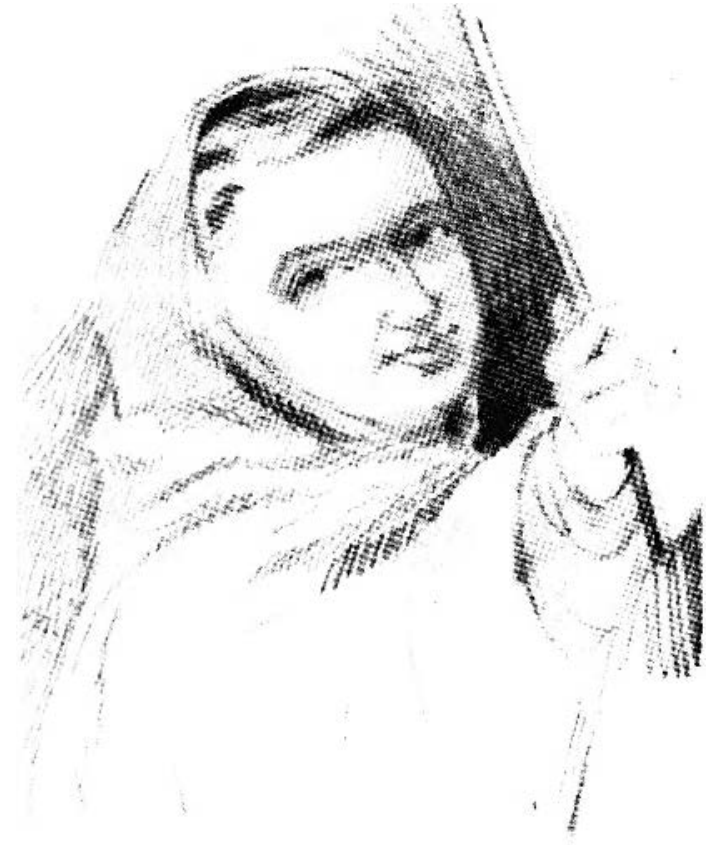

Afortset 39

Figure 5. Gérard Morisset, d'après Claude Françoıs (dit frère I uc), détail de l'Ex-voto à Notre-Dame de Foy, 1932 33. (crédit photographique: dans Gérard Morısset, Lo vie et l'œuvre du frère Luc, Québec. Médium, 1944. planche I)

tieux, bienveillant et discret : visage massif, hautement coloré, empreint d'unc goguenardise paysanne; nez long, épaté, fureteur; lèvres fortement ourlées et gourmandes; grands yeux sombres et vifs. Mélange de traits populaires et de raffinement provincial; sérénité d'un esprit que n'altèrent point les prćoccupations de l'existence quotidienne; joie de vivre; ardeur au travail prestement cxćcuté; c'est avec ces qualités que le Récollet a pcint, parmi des œuvres simplement honorables, des morceaux d'une émouvante ćloquence et d'une tenue digne des maîtres du XVIIe siècle ${ }^{36}$.

Bien que le frère Luc ne soit pas Canadien français et que son séjour en Nouvelle-lirance fut relativement court, il n'empêche qu'il appartient à la " race " des ancêtres (pour reprendre une terminologie utilisée par un idéologue comme Lionel Groulx), ce qui suffit pour que Morisset l'affuble des mêmes qualificatifs. Comme nous l'avons dit, le visage du frère Luc ne suggère pas une lecture psychologique aussi joviale. La détermination de Morisset à présenter le personnage tel qu'il le veut et non tel qu'on le voit s'exprime dans une copie de ce portrait qu'il a lui-même crayonnée (publiée dans La vie et l'auvre du frère Luc, 1944). Les yeux y sont plus rieurs; la bouche y est légèrement plus recourbée, esquissant ainsi un véritable sourire (comparez l'original en fig. 4 avec le dessin de Morisset en fig. 5). Il nous semble que Morisset le dessinateur trahit Morisset l'historien en laissant paraître sur le papier cette manière qu'il a d'améliorer la réalité qui paraît devant son œil.

Et s'il reconnaît que les modèles ne sourient pas toujours, il leur prête malgré tout l'intention de vouloir le faire. On le constate dans sa description du portrait de monseigneur JeanFrançois Hubert par Louis-Chrétien de Heer (fig. 6) : " Le prélat est vu de face; il a une figure ronde, une figure de bourgeois qui voudrait sourire; [... $]^{37}$. " Morisset va encore plus loin avec le portrait de la sœur Sainte-Anne par Antoine Plamondon : "Sur son portrait, elle est sérieuse, ses yeux sont fixes et ses lèvres sont pincćcs comme si la novice réprimait son sourire ${ }^{38}$. " Il voit des sourires où il n'y en a pas, projette des fantasmes de mines réjouies.

À travers son histoire du portrait, qu'il décrive le travail des portraitistes ou les visages de ce peuple qui pose devant le chevalet du peintre, Morisset valorise donc coûte que coûte certaines qualités morales du peuple canadien-français en y associant non seulement l'honnêteté et la bonhomie, mais aussi la vaillance et la bienveillance ${ }^{39}$. Maurice Lemire avait déjà remarqué que, pour les œuvres canadiennes " qu'il estime vraiment, Morisset élabore un discours plein de résonances affectives 40 " (nous l'avons vu tout au long de l'analyse) dont ne bénéficiaient pas les œuvres réalisées par des étrangers. Par exemple, il attribue aux Anglais cette même honnêteté, mais de manière beaucoup plus tiède que pour les peintres canadiens-français ou même les Français "naturalisés ", comme Louis Dulongpré. Les résonances affectives n'interviennent plus. Et en ce qui concerne la gaîté, Morisset la leur refuse, voulant certainement en garder l'exclusivité pour son peuple :

Avec Cockburn, Stewart, Duncan, James Bartlett, BellSmith et combien d'autres, l'art anglais se perpétue au BasCanada, correct et rigide. Portraitistes et paysagistes luttent de conscience et d'exactitude, comme de bons photographes qui cherchent à reproduire de leur mieux les traits des personnages posant devant leurs objectifs. Peu de notes gaies dans l'œuvre immense et froid de ces dessinateurs et peintres, encore moins de fantaisie ${ }^{41}$.

Les stćréotypes nationaux sont délibérément appelés à conforter le propos et, sur ce point, les Allemands sont moins fortunés que les Anglais : "Peinture généralement honnête mais froidc, un peu ennuyeuse par l'abus des symboles, la sécheresse du dessin et la rigidité des vêtements; peinture sans chaleur, sans sourire ${ }^{42}$. "Contrairement à la bonne humeur, l'honnêteté du portraitiste ne semble plus autant le privilège des Canadiens français comme Morisset semblait le suggérer ailleurs. 
Ce n'est d'ailleurs pas la seule contradiction qui habite le discours de Gérard Morisset sur le portrait. Nous avons déjà mentionné les sourires fantasmés de monseigneur Jean-François Hubert, de la sœur Sainte-Anne ou du frère Luc. Nous pourrions ajouter la confiance maintes fois réitérée en la fidélité aux traits des commanditaires, ou le faire vrai, qui demeure invérifiable pour des personnes décédées depuis longtemps (pensons à l'exemple de Champlain discuté plus haut). Enfin, alors même qu'il déplore la fin de l'histoire du portrait au Québec, Morisset avoue qu'une certainc " esquisse de portrait de femme par Borduas " compte parmi lcs trois effigies du XXe siècle auxquelles il prédit la postérité43. Même prise en tant qu'esquisse, cette œuvre est loin d'afficher une minutieuse fidélité aux traits de son modèle; il pourrait s'agir en effet de n'importe quelle femme des années quarante. Que Morisset fasse une exception et se laisse séduire par une œuvre non conforme à son idćal n'a rien de particulièrement paradoxal, mais qu'il la considère comme l'un des trois portraits appelés à survivre à l'histoire l'est beaucoup plus.

Gérard Bouchard, dans La pensée impuissante : échecs et mythes nationaux canadiens-français (1850-1960), affirme que toute entreprise discursive se heurte à des contradictions, des apories : "Dans lc discours (ou la pensée), la raison essaie donc de surmonter les contradictions en faisant intervenir des mythes, en mettant en œuvre diverses stratégies symboliques. Ce faisant, il arrive parfois qu'elle triche en ayant rccours à des falsifications. Alors, elle omet, elle occulte, clle nie, elle réduit ou amplifie, cllc invente ou déforme ${ }^{44}$. "Bouchard l'a bien montré : les idéologues de la période comprise entre l'échec des Patriotes et la Révolution tranquille n'ont pas réussi et parfois n'ont rien fait pour surmonter ces contradictions. Cette incapacité est le propre de ce qu'il appelle la pensée fragmentaire. Lauteur a remarqué que les penseurs importants de certe période ne semblent mêmc pas se rendre compte de leurs contradictions : "Tout se passe comme si le discours ne gardait pas la mémoire de ses incohérences [... $]^{45}$ ". Gérard Morisser participe de cette pensée fragmentaire qui caractérise ses contemporains. Il rejoint, mais dans le champ de l'histoire de l'art, les Lionel Groulx, Arthur Buies, Edmond de Nevers, Édouard Montpetit et Jean-Charles Harvey dont Gérard Bouchard a disséqué les discours.

Au fond, les paradoxes de Morisset sont les symptômes de l'idéologic, de la pensée impuissante de son temps. En occultant les contradictions et les incohérences, il utilise son discours sur le portrait pour nourrir un autre portrait-nationaliste celui-là-où le Canadien français est dépeint à tout prix comme un travailleur de bonne humeur, consciencieux et honnête. Ces qualités doivent se concrétiser-voire se vérifierdans les œuvres d'art. Et si le mythe ne correspond pas à la peinture, ne serait-ce qu'en apparence, Morisset se persuade du contraire. Ainsi, l'idéologie nationaliste dans laquellc s'inscrit

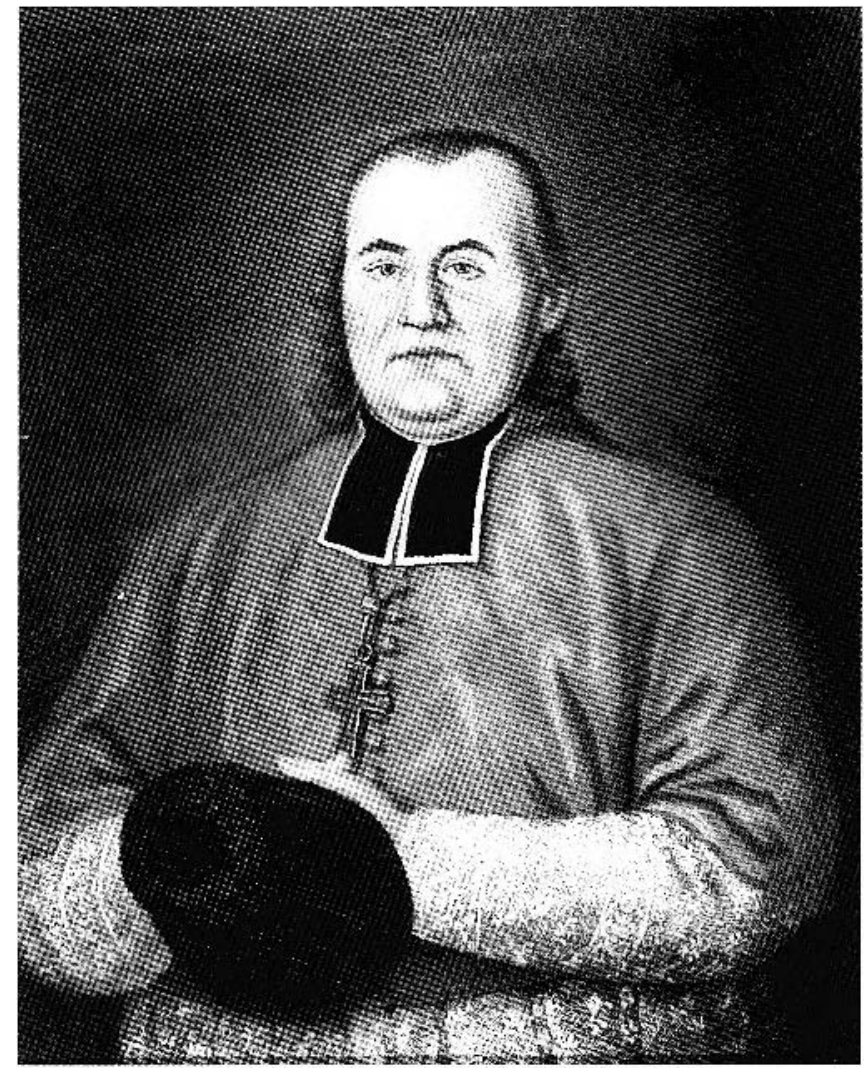

Figure 6. Louis-Chrétien de Heer (attribué à). Portroit de Mgr Jean-François Hubert, 1788, huile sur tolle, $81.4 \times 66 \mathrm{~cm}$. Québec, Musée de la civilisation, collection de l'Archevêché de Qućbec (crédit photographique : Idra Labrie Perspective, N 1991.3875).

Gérard Morisset engendre plusieurs points aveugles qui font que deux idées contradictoires peuvent coexister en s'ignorant, mais de ce fait, s'affaiblissent et se discréditent l'une l'autre. Bien entendu, toutcs les idéologies occasionnent un certain degré de cécité intellectuelle, mais les plus fortes réussissent néanmoins à maquiller les contradictions, soit en acceptant un compromis entre les deux termes incompatibles, soit en rejetant l'un de ces deux termes, mais jamais en ignorant l'incohérence ${ }^{46}$. Maintenant que nous avons expliqué comment l'histoire du portrait construite par Morisset a joué un rôle de valorisation, au moyen de quels mécanismes et avec quelles contradictions, il nous reste encore à tenter de comprendre ses motivations, autrement dit à nous intéresser à celui qui, caché derrière l'historien et l'auteur, fut un homme de son temps. 


\section{Morisset, historien de l'art : un homme de son temps}

Dans ses Douze leçons d'histoire, Antoine Prost partageait avec son lecteur une réflexion qui s'avère pertinente pour une étude historiographique telle que la nôtre :

[...] l'histoire des questions historiques n'est pas seulement I'histoire, scientifique et sociale, des " écoles " historiques. Flle n'obéit pas seulement à des facteurs internes à la profession. Celle-ci est en effet globalement insérée dans unc société pour laquelle elle fonctionne er qui la fait vivre. Ellc cst d'autre parr composée d'individus qui ont chacun des raisons personnelles de faire l'histoire. La question historique est posée non sculcment au sein d'une profession, mais au sein d'unc société ct par des personnes. Double polarité qu'il nous faut $\operatorname{cxplorcr}^{47}$.

Prost distingue l'enracinement personnel et l'enracinement social des questions historiques qui sont posées par les historiens. Les écrits de Gérard Morisset sur l'art du portrait au Canada français montrent que, loin d'être enfoui, ce double enracinement conditionne et motive l'essentiel du projet historique morissetien.

Enracinement personnel des questions historiques

de Gérard Morisset

Selon Prost, il faut toujours garder à l'esprit le poids de la personnalité de l'historien lorsqu'il écrit, car cclui-ci " noue avec son objet une relation intime, où s'affirme progressivement sa propre identité. En se penchant sur la vic ct la mort des hommes du passé, il travaille aussi sur sa propre vie et sa propre mort 48 ". Déjà en 1981, les esquisses biographiques contenues dans l'ouvrage collectif $\dot{A}$ la découverte du patrimoine avec Gérard Morisset 49 nous permettaient d'approfondir cette dimension personnelle si importante dans les textes de Morisset. Le présent essai s'appuiera fortement sur ces efforts antérieurs pour éclairer les différents points d'analysc de la section précédente.

Posons d'emblée l'élément biographique le plus significatif : Gérard Morisset était Canadien français et adhérait complètement à cette identité nationale. Non seulement écrivait-il en tant que Canadien français, mais encore s'adressait-il à des lecteurs qui partageaient la même identité. L'usage constant, à travers son œuvre écrit, des pronoms (personnel et possessifs) de la première personne du pluriel en témoigne clairement ; il emploie fréquemment le " nous " pour désigner le peuple auquel il appartient et il n'est pas rare de lire les cxpressions " notre passé " ou " nos ancêtres " 50 .

Né en 1898 à Cap-Santé, donc en milieu rural, Morissct est issu d'une famille relativement humble. Il était le fils d'un artisan-ferblantier. Bien que ses études europécnncs l'aient élevé à un statut supérieur à celui auquel il était destiné, on sent chez Morisset un désir de rester fidèle à la tradition que représente son père. En témoigne son attachement à certaines valeurs tributaires de l'éducation paternelle, telles l'honnêteté professionnelle ou l'amour de la "bclle ouvraige bian faittes1 ", qui expliquerait en partie pourquoi il valorisait tant l'honnêteté et l'éthique professionnelle des portraitistes recherchant laborieusement la ressemblance du client, ainsi que lcur méticulosité à rendre les détails.

Ainsi, son analyse de la façon dont les traditions artisanales ont survécu après la chute du régime français est clairement imprégnée du souvenir laissé par l'exemple d'un pìre artisan :

Et le traité de Paris, loin de tarir l'inspiration et l'activité de ses artisans, leur a fourni une raison de plus de rester cux-mêmes et de vivre au Canada la même existence qu'ils auraient vécue sans l'infortune militaire de la guerre de Sept Ans.

Ces fortes traditions, nos artisans les ont maintenues en peinture; avec moins de brio que dans les autres arts, pour les raisons que j'ai dites; mais avec continuité, avec cette douce tyrannie de la chose bien faite ${ }^{52}$.

N'oublions pas que Gérard Morisset fut également thćoricien de l'architecture (et même clandestinement architecte) avant de séjourner en France. Il possédait déjà cn matière de construction une conception particulière de l'honnêtcté qui devait s'exprimer soit par l'utilisation appropriée des matériaux, soit par une attitude réaliste et logique dans la conception d'un bâtiment, c'està-dire par une conception rationaliste qui nc laissc pas de place au pastiche ou au caprice 53 .

Sa conception de l'artiste ou de l'artisan canadien-français, voire du Canadien français en général, vaillant au travail et toujours consciencieux, ne peut pas non plus être séparée du rapport que Morisset entretenait avec le travail. On connaît son acharnement dans la constitution de l'Inventaire des CEuvres d'Art de la Province à laquelle il travailla pleinement à partir de 1937. Le nom de cet inventaire en dit long sur l'ambition même de Gérard Morisset. Les milliers de fiches qu'il a complétées pour inventorier et documenter les œuvres d'art reflètent la minutie d'un homme qui se plaît à retrouver cctte même qualité chez les artisans dont il se considère le descendant. En faisant l'énumération de tous les accomplissements de Gérard Morisset au cours de sa vie-ses centaines d'articles et de conférences, ses livres et son roman, son projet d'inventaire, ses grandes charges gouvernementales (Inspecteur de l'enseignement du dessin dans la Province de Québec, Secrétaire à la commission des Monuments Historiques du Québec et Conservateur du Musée de la Province), on ne peut douter qu'il ait lui-mĉme ćté un bourreau de travail. À la lumière de ces éléments biographiques, le portrait qu'il nous a laissé de François Baillairgé s'interprète tout 
différemment : " Habile et consciencieux, bon vivant, facétieux, humble mais conscient de sa valeur, il a travaillé toute sa vie avec une bonne humeur et un entrain qui percent à chaque page de son Journal 54. "Que toutes ces qualités puissent effectivement se dégager du Journal de Baillairgé (qui est d'abord et avant tout un livre de raison, c'est-à-dire un livre de comptes), il est aventureux de le confirmer tant celles-ci semblent relever de la projection.

En ce qui regarde sa conception de la bonhomie et de la simplicité, peut-être vient-elle du regard qu'il posait lui-même sur ses origines rurales. Du moins, c'est ce que tend à nous faire croire sa description de Ludger Ruelland, "portraitiste d'une fine probité " : "Fils d'un humble villageois, il passe son existence à peindrc des petites gens, citadins ct bourgeois de campagne, ecclésiastiques et négociants; et lorsque le hasard amène devant son chcvalet un personnage important, il le voit avec ses yeux de villageois et le peint dans sa condition primitive. D'où une bonhomie constante dans son œuvre, une simplicité aimables5. "Les "yeux de villageois " de Ludger Ruelland scraient responsables de la bonhomie constante qui règne dans son œuvre. Nous croyons pouvoir en dire autant du regard que Gérard Morisset posait sur les portraits et les artistes du passé de son pays.

Son éducation classique lui a permis d'accéder au notariat qui représentait l'un des trois secteurs propres à l'élite francophone traditionnelle. Morisset a également bénéficié d'un certain état d'esprit au Québec, qui a voulu encourager la société à sc constituer une élice intellcctuelle capable d'agir en dchors de l'indépassable trio médecinc-droit-prêtrise. De fait, Morisset profita de bourses gouvernementales, à l'instar de quelques compatriotes célc̀bres, pour aller se perfectionner en France. Il avait donc conscience du rôle important qu'on espérait qu'il joue dans la socićté canadienne-française à son retour. De par sa formation et de par le statut qu'elle lui procurait, Morisset érait donc disposé à adhérer à ccrtaines positions exprimées par les milieux intellectuels quant à la sauvegarde du passéś. Serge Gagnon écrivait à ce propos :

La formation sociale dans laquelle l'historien évolue lui imprime une vision du monde articulée à l'expćrience de la société globale à laquelle il appartient. Au sein de certe totalité, la place qu'il occupe dans la hiérarchie sociale permet d'expliquer scs choix, ses jugements de valeurs, implicites ou explicites, $[\ldots]$ ses silences aussi bien que scs généralisations à l'égard de tel ou tel aspect de la réalité sociale ${ }^{57}$.

L'enracinement personnel sc confondant souvent avec l'enracinement social, il est préférable de réserver les conséquences de ce faic pour la prochaine section.

Parti à l'origine pour acquérir plus d'expérience en matière d'architecture, Morisset bifurqua vers la prestigieuse École du Louvre pour y étudier une histoire de l'art à la française, em- preinte de connoisseurship et de traditions rhétoriques propres. Cette formation détermina fortement son approche de l'art ${ }^{58}$. Pensons par exemple à la lecture physiognomoniste du portrait qui marquait depuis plusieurs sic̀cles les écrits sur le portrait. C'est également grâce à cette formation qu'il a eu le grand mérite d'être le premier au Québec à porter un véritable discours d'historien de l'art sur le portrait canadien-français.

Enfin, il faut mentionner sa longue expérience de chroniqueur et de journaliste, que ce soit dans la pressc écrite ou à la radio. Plusieurs de ses articles de journaux ont servi à nos analyses er plusieurs de ses livres sont eux-mêmes la synthèse de chroniques antérieures. Il n'est d'ailleurs pas rare de le voir repiquer ou môme copier intégralement dans ses ouvrages une phrase, un paragraphe tiré d'un ancien article. Sa lecture du portrait conserve quelque chose du ton journalistique, des formulations, de la rhétorique de la description er de l'énumération propres à la presse écrite.

Enracinement social des questıons historiques de Gérard Morisset Antoine Prost attire l'attention de ses lecteurs sur le fait que " toute question historique, en effet, est posće hic et nunc, par un homme situé dans unc socićté. Quand bien même il veut lui tourner le dos et donncr à l'histoire une fonction de pure connaissance désintéressée, il ne peut fairc qu'il ne soit de son temps 59 ". Ainsi, ces questions " préscntent généralement un intérêt pour la sociétć au sein de laquelle elles sont posées $^{(0)} \%$. Morisset est loin d'échapper à la rc̀gle. Comme nous l'avons déjà dit, par sa formation et par la place qu'il occupait dans la socićtć, Morisset était tout disposé à adhérer à certaines idées de l'ćlite intellectuelle dont il faisait partie. Le contexte du nationalisme d'avant lcs années soixante recèle précisément les bases idéologiques sur lcsquelles repose le discours de Morisset.

\section{Le nationalisme canadien-français (1840-1960)}

Le courant de pensée dans lequel s'inscrit Gérard Morisset est celui d'un nationalisme de conservation ${ }^{61}$, ce que Louis Balthazar a nommé la période du nationalisme canadien-français, débutant avcc la fusion du Haut-Canada et du Bas-Canada sanctionnée par l'Acte d'Union (1841) et se terminant avec la Révolution tranquille dans les années $1960^{62}$. Ni moderne ni étatiste (pour reprendre les distinctions dc Louis Balthazar), mais plutôt traditionnel, ce type de nationalisme s'enracinc dans l'histoire et le passé et maintient une certaine distance vis-à-vis du pouvoir et du politique (ce que nuance Gérard Bouchard(3), se repliant plutôt du côté de la culture et de sa préservation ${ }^{64}$.

Durant cette période, les élites s'emploient à construire une identité proprement canadienne-française. Parmi les nombreux moyens employés pour y parvenir, il y eut le recours à 
l'histoire pour bâtir une mémoire nationale, pour se réapproprier le passé et la culture des ancêtres65. Comme le note Gérard Bouchard :

\begin{abstract}
À partir du milieu du XIXe siècle surtout (il y eut toutefois des initiatives dans ce sens dès le début du siècle), un très grand nombre de lcttrés [...] oeuvrèrent à [la] promotion [de la culture populaire] d'une manière ou d'une aurre : collecte de données orales, publication de répertoires, conservation d'artefacts, rédaction de contes et de légendes. Officiellement, il s'agissait de faire œuvre de civilisation en assurant la mémoire de ce précieux matériau symbolique avant qu'il ne disparaisse 66 .
\end{abstract}

Morisset prolonge cette attitude de réappropriation du passé en aménageant une place au patrimoine artistique. Il est tout à fait conscient que ce genre de travail advient un peu tard par rapport à ce qui s'est déjà fait dans les domaines de l'histoire événementielle, de la littérature et de l'ethnographie : "En général, nos historiens ont ignoré l'art-cette manifestation collective et spontanée d'un peuple sensible et sain, dont le langage et les formes plastiques, les objets usuels et d'agrément jouent un rôle primordial dans l'évolution de sa culture. Le Rapport de Durham leur a peut-être enlevé toute envie d'y aller voir-et c'est regrettable ${ }^{67}$."

La peinture traditionnelle au Canada français se lit comme une réplique au célèbre Rapport Durham, faisant écho, un siècle plus tard, à l'Histoire du Canada de François-Xavier Garneau ${ }^{68}$. Les passages qui ont le plus fait réagir les intellectuels et les historiens canadiens-français_et ce, bien avant ct bien après l'époque de Morisset-sont sans doute ceux où Durham écrivait : "Ils sont un peuple sans histoire et sans littérature [...] " ou "Leur nationalité a pour effet de les priver des plaisirs et de l'influence civilisatrice des arts ${ }^{69}$. " En effet, aussi tôt qu'en 1936, Morisset y fit une subtile allusion alors qu'il tâchait de justifier la pertinence sociale de son travail :

Mais encorc par souci intellectuel; par respect pour la mémoire de nos pères qui, soit dit en passant, nous valaient bien; pour montrer aux autres que nous ne sommes pas une tribu nomade, un troupeau de hordes dénuées de tout sens du beau, sans aspirations comme sans passé; pour nous prouver à nous-mêmes que nos ascendants n'ont pas lutté en vain pour nous léguer un peu de culture ${ }^{70}$.

Toutefois, Morisset ne fut pas le premier à vouloir réintégrer le patrimoine artistique à la mémoire nationale. Ainsi, quelques années avant lui, Georges Bellerive posait le problème dans les mêmes termes dans son ouvrage Artistes-peintres canadiensfrançais (1927). L'auteur commence son livre en reproduisant un passage d'un discours livré en 1922 par l'Honorable Athanase David, Secrétaire de la province, qu'on surnommait alors le "Mécène de la Province " en raison de son implication dans le développement de la culture au Québec : "Aimons, admirons tous ceux qui dans les divers domaines firent leur part du devoir ; ce n'est pas seulement du patriotisme de bon aloi, mais aussi un signe de bonne santé. En effet, la nation qui sait aimer et admirer n'est pas près de mourir ${ }^{71}$. " Athanase David, qui financera en premicr Gérard Morisset pour la constitution de l'Inventaire des CEuvres d'Art, fait notamment référence aux peintres du passé. Il n'est pas fortuit que Bellerive ait choisi de citer le "Mécène de la Province " dans la préface de son ouvrage. Cela témoigne, en fait, de la portée nationaliste des initiatives de réappropriation du passé artistique dont font partie celle de Morisset ou encorc celle de Marius Barbeau.

Les entreprises historiques ou littéraires des élites qui ont réagi au rapport Durham à partir des années 1840 , tout en construisant la mémoire et l'identité nationales, ont tenté de dépeindre l'" âme canadienne-française " sous son jour le plus favorable. Comme nous l'avons vu, Morisset ne se distingue pas en cela des autres. L'abbé H.-R. Casgrain incitait d'ailleurs les écrivains à décrire lc peuple " non pas tel qu'il est mais tel qu'on lui propose d'être 72 ". C'est exactement ce que fit Morisset en présentant un artiste ou une personne portraiturée.

Gérard Bouchard notait qu'à travers les diverses tentatives qui ont été menées en ce sens, on retrouvait un " éventail variable de valeurs et de qualités morales [qui] complétaient l' "âme " canadienne-française : l'orientation spirituelle, l'esprit communautaire, la modestie, la vaillance, l'intégrité, le sens de la hiérarchic ${ }^{73}$ ". Nous avons vu chez Morissct les qualités de vaillance et d'intégrité, mais si nous avions analysé d'autres discours que celui qu'il tient sur le portrait, nous y aurions aussi retrouvé la modestie et l'esprit communautaire. Le patriotisme de Gérard Morisset, et cela peut nous surprendre, ne fait toutefois pas grand cas des trois piliers majeurs qui vont caractériser une idéologie commc le clérico-nationalisme, à savoir la langue française, la religion catholique et la famille. Qu'il se distingue en ceci ne l'empêchera cependant pas d'aller puiser certaines conceptions dans la pensée de Lionel Groulx.

\section{Lionel Groulx et la « race » canadienne-française}

C'est effectivement chez cet historien et idéologue qu'il faut chercher des indices pour nous aider à comprendre les écrits de Morisset, son contemporain. Celui que l'historiographie considère comme le nouveau maître à penser du nationalisme canadien-français va jouir à partir de 1920 d'une influence qui, bien que moins forte après 1945, s'étendra néanmoins aux années soixante.

Son idéal mythique du Canadien français repose sur un concept de " race " qu'on aurait tort d'interpréter à la lumière des significations contemporaines. Comme l'a déjà fait remar- 
quer Louis Balthazar, ce mot réfere davantage chez Groulx à ce qu'on appellerait aujourd'hui " ethnie ", bien que la dimension héréditaire et le "sang des aïeux " y prennent une place prépondérante ${ }^{74}$. Il n'en demcure pas moins que l'idéologie derrière le mot est souvent trouble et nécessite une remisc $\mathrm{cn}$ contexte extrêmement prudente ${ }^{75}$. L'héréditaire, chez Groulx, peut comprendre non seulement des caractéristiques physiques, mais aussi des qualités ou des tares morales. Jean-Pierre Gaboury décèle dans cette conception l'influence des théories de Gustave Le Bon qu'on peut retrouver dans les Lois psychologiques de l'cvolution des peuples (1919)76. Voici un extrait que l'abbé avait souligné dans son propre exemplaire : "Cclleci [la psychologie moderne] montre que, derrière les institutions, les arts, les croyances, les bouleversements politiques de chaque peuple, se trouvent certains caractères moraux et intellectuels dont son évolution dérive. C'est l'ensemble de ces caractèrcs qui forme ce que l'on peut appeler l'âme d'une race 77 ."

Dans son ouvrage La naissance d'une race (1938), Lionel Groulx expose une théorie évolutionniste selon laquelle la " race canadienne-française "-héritière de certains traits des ancêtres brctons, normands, etc. - se scrait développée en réagissant à certains facteurs climatiques et géographiques, mais aussi à la nature des institutions politiques et religieuses de la NouvclleFrance. D'où une panoplie de qualités et de défauts qui constituent la singularité de cette " race nouvelle ", expression que Groulx emploie pour titrer le chapitre conclusif de son ouvrage. Il tâche d'excuser un certain goût pour l'alcool, pour la vantardise et pour la vanité dans l'habillement par des traits supposément distinctifs : robustesse et beauté physiques, esprit libre et aventurier, couragc, endurance, esprit de coopćration 78 . Et parmi cette liste tout de même limitée, s'ajoutent encore quelques traits de caractère également prisés par Morisset.

Il y a d'abord la probitć du peuple canadien-français, ce qui ne nous surprend guc̀rc (Lionel Groulx tente de donner plus de crédibilité à l'ancienneté de cette vertu en citant des chroniqueurs d'Ancien Régime comme le père Charlevoix $\left.{ }^{79}\right)$. Il y a aussi son ardeur au travail, son degré d'habilcté manuelle, son ingéniosité mécanique et son inclination pour les arts ${ }^{80}$. Et enfin, nous l'aurons deviné sans peine, il y a sa gaîté naturelle. Dans le portrait qu'il trace des aïeux tout au long de son panégyrique intitulé Chez nos ancêtres (1920), Groulx écrit : "La vie canadienne de ce temps-là si unie, si enclose peut paraître monotone, ennuyeusc. Pourtant comme nos ancêtres étaient gais, d'une gaieté franche, $[\ldots]^{81}$. " Dans La naissance d'une race (1938), l'auteur développe plus rigoureusement, mais plus longuement aussi, son argumentaire sur la gaîté canadienne-française. En voici un extrait : "L'endurance canadienne a du rcste un autre appui : celui d'une gaîté franche et bien française, de la meilleure qualité. On n'eût point trou- vé chez eux cette "conception du monde propre aux peuples du nord, toute triste et toute morale." Même aux plus mauvais jours de leur histoire, nos gais aïeux savent rire, chanter et même chansonner ${ }^{82}$."

Ainsi, il appert clairement que le discours de Morisset sur le portrait est teinté par certains aspects du discours idéologique nationaliste de son époque. De la même manière que les romanciers et les historiens de la période 1840-1960 ont tâché de dépeindre, à travcrs leurs écrits, l'âme (idéalisée) du peuple canadien-français—voire de la " race " canadienne-française-, ainsi Morisset parvient-il, par des propos historiques sur un genre pictural tel que le portrait, à construire une sorte d'idéal nationaliste du Canadien français, qui se nourrit inévitablement des représentations véhiculées par l'imaginaire collectif. L'approche dc Cérard Morisset est toutefois différente de celle de Lionel Groulx : alors que ce dernier valorise le peuple en général et attribue des qualités morales à une collectivitć, Morisset, quant à lui, distribue ces mêmes qualités à des individus qui deviennent autant de figures de cas exemplifiant l'idéal qu'il partage avec Groulx, autant d'avatars de ce "nous " canadien-français.

À force d'enthousiasme, Morisset (comme Groulx d'ailleurs) améliore la réalité, véhicule une ccrtaine forme de propagande. Sur la proximité qui existe entre l'histoire et l'idéologie, Serge Gagnon écrivait : "'lhe collective memory simplifies and distorts. Its role is to stimulate a sense of belonging, of social solidarity. It carries an intense emotional charge designed to bring the members of a community together around certain symbols 83 . "Cette charge émotionnelle transparaît à travers les outils qui sont propres à l'historien de l'art. L'analyse formellc, par exemple, qui dans l'idée passe pour la plus « objective", peut en être le véhicule comme n'importe quel autre outil d'analyse. À propos de l'historiographie des arts canadiens, Jacques-Gilles Fournier relève qu'il y a

chcz les historiens ou historiographes [...] des a priori secrets $|\ldots|$. Lanalysc formelle autorise chez ces historiographes, qu'ils soient canadiens français ou anglais, des jugcments de valeurs sur la société dont ils n'inventoriaient au départ que Ics témoins culturcls. Il y a chez cux un besoin de déplacer les valeurs. Les valeurs esthétiques des témoins culturels se métamorphosent souvent en "valeurs raciques 84 ".

Effectivement, c'est le plus souvent sous le couvert de l'analyse formelle que Morisset trahit ses partis pris. Dans la minutie d'un coup de pinceau pouvait se déceler l'honnĉteté d'un portraitiste canadien-français, dans les lignes curvilignes formant l'image d'une bouche, "le sourirc de la race". 


\section{Conclusion}

Le mythe ne meurt pas avec Morisset. On peut encore en déceler les traces dans une monographie sur Roy-Audy publiée par Michel Cauchon en 1971. Lauteur commente ainsi l'œuvre du portraitiste : "Cette préoccupation pour la ressemblance est caractéristique de l'œuvre et même de la clientèle de RoyAudy. Ce dernier conserve sans doute au fond de son cœur cette honnêteté professionnelle de l'artisan pour qui le travail bien fait est un point d'honneur ${ }^{85}$. "En décrivant ses portraits, il écrit : "Dès le début de sa carrière en 1823, Roy-Audy parvenait à rendre l'austérité et le raffinement germaniques du docteur Charles Auguste Globensky pendant qu'il saisissait un regard volontairement sévère dans le visage empreint de bonté de son épouse canadienne-française 86 . "On relève effectivement dans ces deux passages l'essentiel des préjugés morissetiens. La relation qu'a entretenuc la communauté des chercheurs en histoire de l'art québécois avec son fondateur reste encore à défricher. Cet exemple isolé ne peut rendre compte de l'influence quc Morisset a eue sur ses héritiers, mais il prouve néanmoins qu'il y a matière à analyse.

Nous nous sommes efforcés jusqu'ici d'identifier les causes expliquant les partis pris de Gérard Morisset, puisant divers éléments dans sa vie et dans le contexte idéologique qui fut le sien. En privilégiant les causes, peut-ĉtre avonsnous négligé les fins. Aussi, pour conclure, convicnt-il de dire quelques mots sur les intentions qui sous-tendaient ses analyses parfois biaisées.

L'auteur semblait croire que l'exemple des ancêtres et de la tradition pouvait être une source d'ćdification, sinon d'émulation, à une époque de déclin : " Je cherche uniqucment à faire réfléchir mes lecteurs sur la vie profondément humaine de nos pères, sur lc moteur de leur force morale, sur les causes de notre décadence ${ }^{87}$. "Ces causes, il les identifie comme ćtant "l'affaissement de la vie intérieure, la disparition de la simplicité, la ruine des traditions artisanales 88 ". Pour Morisset, la société qui est la sienne a rompu avec le passé89, elle ne comprend plus ses ancêtres 90 cr cllc a renié les valeurs traditionnelles qui ont fait qu' "un ordre social sainement équilibréyl " a régné en Nouvelle-France. De là, il s'ensuit que la société du XXe siècle n'arrive plus à être en santé, donc à se préserver.

Parce qu'il est le reflet d'un peuple sain, l'art traditionnel (et particulièrement le portrait) est pour Morisset le meilleur moyen qu'il ait trouvé pour édifier ses contemporains:

À son tour, le peuple lui communique [à l'art] son esprit, son caractère, sa bonhomie, même son aimable jovialité. Longtemps notre peinture a été fonctionnelle et socialc, comme nos autres arts d'ailleurs. Voilà pourquoi elle nous aide à comprendre notre passé, à déchiffrer l'âme de ces bourgeois et de ces paysans qui avaient moins que nous le sens du drame et plus que nous le sens de la vie lente et de la véritable gravité 92 .

Morisset s'est efforcé par sa critique du portrait de déchiffrer cette " âme canadienne-française " qu'il aurait voulu voir se perpétuer jusqu'à son époque. "Notre sentimentalité et, disons le mot, notre vanité collective y perdront des adeptes, bien sûr : en revanche, le patriotisme véritable y gagnera. Car on n'aime d'un amour profond que les êtres qu'on connaît bien ${ }^{33}$. " Peut-être faisait-il écho à cette parole de l'honorable Athanase David qu'il a lue chez Bellerive ou qu'il a peut-être entendue de la bouche même du Secrétaire de la province : " [...] la nation qui sait aimer et admirer n'est pas près de mourir ${ }^{44}$." "

Ainsi, son discours sur le portrait ne se résume pas à une campagne de valorisation et d'auto-congratulation qui aurait pour but de montrer à quel point le Canadien français est digne de louange. Il se veut un baume, à défaut d'être un remède, contre ce qu'il perçoit comme la décadence du XXe siècle. Il n'est pas rarc que l'historien porte une plus grande attention à ce qui-dans lc passé-peut apporter une solution aux problèmes du présent ${ }^{95}$. La manière dont Gérard Morisset entrevoyait la pertinence socialc de son travail doit nous rappelcr que la vision de notre propre pertinence en tant qu'historienne ou historien interfere toujours avec la notion illusoire d'une histoire " purement " désintéressée.

\section{Remerciements}

Je tiens à remercier Monsieur Laurier Lacroix de m'avoir inspiré ce projet et surtout de m'avoir communiqué son grand intérêt pour l'étude de l'historiographie de l'art au Québec. Merci également à l'évaluateur anonyme de la revue dont les remarques très éclairantes ont contribué à l'amélioration du texte. La diligence et la générosité de Soeur Marie-Berthe Bailly, Madame Lucie K. Morisset, Monsieur Jacques Saint-Arnaud et Monsieur Alain Dulin ont facilité grandement la publication des illustrations.

\section{Notes}

1 Gérard Morissct, "Le peintre François Beaucourt ", La Patrie, 19 mars 1950, p. 50.

2 Ayant reçu une formation à l'École du Louvre dans les années trente, Gérard Morissct revint dans la province pour étudier, valoriser et faire connaître à tous les publics le patrimoine artistique du Québec qui n’avait reçu jusque-là qu'une médiocre attention, d'ailleurs souvent teintée de dédain. C'est à lui que l'on doit l'initiative, en 1937, de l'Inventaire des CEuvres d'Art dont la mission ambitieuse était de recenser le plus exhaustivement possible les œeuvres d'architecture, de peinture, de sculprure et d'orfevrerie produites sur le territoire de la province. 
3 Gérard Morisset, Coup d'ceil sur les arts en Nouvelle-France, Québec, à comptc d'auteur, 1941, p. 72-3.

4 lbid., p. 129-48.

5 Il explique la fin de l'histoire du portrait par l'avènement de la photographie, lc costume sévère de son temps, l'indifférence du public et les prix exigés par les artistes. Gérard Morisset, Portraits canadiens du $18 \mathrm{e}$ et $19 \mathrm{e}$ siècles / Canadian portraits of the 18th and 19th centuries, catalogue d'exposition, Ottawa ct Québec, Galerie nationale du Canada et Musée de la Province, 1959, n.p.

6 Gérard Morisset, "Le portrait canadien il y a un siècle ", Le Droit, 3 août 1935 , p. 9.

7 Il faut ici payer notre tribut au travail conduit par Robert Derome qui a mis à la disposition des chercheurs les photocopies des articles publiés par Morisset au cours de son existence. Flles se trouvcnt à la réserve de la Bibliothèque des Arts de l'Univcrsité du Québec à Montréal. Une partie de ces textes est disponible en ligne : Robcrt Derome, Gérard Morisset (1898-1970) : recueil de ses écrits, www. cr.uqam.ca/nobel/r14310/Morisset/ [consultć lc 11 février 2008].

8 Société Numismatique et Archéologique de Montréal, Descriptive Catalogue of a Loan Exhibition of Canadian Historical Portraits and other objects relating to Canadian archaeology held in the Natural History Society's building by the Numismatic and Antiquarian Society of Montreal, in commemoration of the 25th anniversary of the foundation of the society on Ihursday, December 15th, 1887, and following days, catalogue d'exposition, Montréal, Gazette Printing Company, 1887, 80 p. ; Archibald Chaussegros de Léry Macdonald, $A$ record of Canadian historical portraits and antiquities exhibited by the Numismatic and Antiquarian Society of Montreal, 15th September 1892 in commemoration of the 250th year of the foundation of Montreal, catalogue d'exposition, Montréal, Numismatic and Antiquarian Society, 1892, 56 p. ; Thomas O'Leary, Catalogue of the Chateau Ramezay Museum and Portrait Gallery, Montréal, Numismatic and Antiquarian Society, 1901, 128 p.

9 Maurice Lemire, "Gérard Morisset et l'écriture ", dans $A$ la découverte du patrimoine avec Gérard Morisset, Qućbec, Ministère des affaires culturelles / Musée du (Québec, 1981, p. 193-203.

10 Gérard Morisset, "Deux jolies pcintures ", le Terroir, vol. XVI, no. 5, octobre 1934, p. 7.

11 Gérard Morisset, Novembre 1775 : nouvelle, Québec, chez l'auteur, 1948 , p. 33.

12 Comme l'a montré Édouard Pommier, l'idée selon laqucllc lc portrait devait révéler " le véritable caractère ct tcmpérament du modèle " fut l'un des lieux communs qui circulaicnt dans les traités, les lettres et les poèmes évoquant l'art du portrait à la Renaissancc, c'est-à-dire à une époque où les théories physiognomonistes étaicnt généralement bien admises. Il va sans dire qu'à partir de ce moment, si ce n'est depuis l'Antiquité, cette conception va contaminer la rhétorique de la description du portrait de manière indélébile. Morisset l'a probablement intégrée dans les années trente pendant sa formation à l'Écolc du Louvre, où l'on enseignait une histoire de l'art à la française tout à fait familière avec ce genre de procédés stylistiques. Pour plus de détails sur la relation entrc physiognomonie et théories du portrait, voir Édouard Pommier, Theories du portrait : de la Renaissance aux Lumières, Paris, Gallimard, 1998, p. 112-16.

13 Gćrard Morisset, Peintres et tableaux : les arts au Canada français, Québec, Éditions du Chevalet, 1936, p. 142.

14 Cérard Morisset, "Les déburs de la peinture en Nouvelle-France : le portrait de Champlain ", Le Canada, 5 juillet 1934, p. 2.

15 Gérard Morisset, Exposition rétrospective de l'art au Canada français / The Arts in French Canada, cataloguc d'exposition, Québec, Musée de la Province ct Secrétariat de la Province, 1952, p. 8. Dans le cadre de ce catalogue, qui devait probablement servir de guide d'exposition étant donné son petit format et sa minceur, on constate que Morisset a tenté d'orienter idéologiquement le regard porté par le visiteur sur les portraits exposés.

16 Ibid.

17 Morisset, Coup d'ail sur les arts en Nouvelle-France, p. IX-X.

18 Nous reviendrons sur la notion dc race et sur ses connotations dans le contexte idéologique des annécs trente. Morisset, "Deux jolics pcintures", p. 8.

19 L'attribution de Morisset a été désavouée. Ce portrait est aujourd'hui attribué à Louis-Chrétien de Hccr. Gérard Morisset, "François Baillairgé (1759-1830) : le peintre ", Technique, vol. XXIII, no. 4, avril 1948, p. 230-31.

20 Morisset, Coup d'xil sur les arts en Nouvelle-France, p. 75.

21 Gérard Morisset, "Un grand portraitiste, Antoine Plamondon ", Concorde, vol. XI, nos. 5-6, mai-juin 1960, p. 14.

22 Gérard Morissct, "Portraits de mortes en Nouvelle-France ", l.e Canada, 25 mars 1935, p. 2.

23 Gérard Morisset, "Un primitif, Jean-Baptiste Roy-Audy : son ceuvrc", Technique, vol. XXVIII, no. 8, octobre 1953, p. 545.

24 Gérard Morisset, "Antoine Plamondon (1804-1895)", Vie des arts, mai-juin 1956, p. 10.

25 Gérard Morisset, La Peinture traditionnelle au Canada français, Ottawa, Le Cercle du livre de Francc, 1960, p. 161.

26 Morisset, "Deux jolies peintures ", p. 8.

27 Morisset, Peintres et tableaux, p. 101. On se souviendra que le mythe de l'École de Saint-Joachim n'avait pas encore été déconstruit. Quant au choix de l'expression " caractère photographiquc" pour décrire l'ocuvrc de Louis Dulongpré (1759-1843), il est aussi étonnant qu'intéressant. Pour mcttre en relief l'honnêteté du portraitiste, sa fidélité dans l'observation du modèle est comparée à ccllc de l'appareil photographiquc. Ici, l'anachronisme tient lieu d'hypcrbole. Paradoxalement, dans Portraits canadiens du 18 et $19 e$ siècles, Morissct dénonce l'avènement de la photographie comme l'une des quatrc causes expliquant la fin de l'histoire du portrait cn plus de se montrer contre l'attitude de copistc servile et sans discernement qu'adoptaient certains peintres d'histoire religieuse. La référence à la photographie est malléable : tantôt elle sert à louer 
la fidélité du portrait, tantôt elle sert à en expliquer le déclin.

28 Canada français, p. 8.

29 Pommier, op. cit., p. 108.

30 Morisset, Exposition rétrospective de l'art au Canada français, p. 8.

31 Morisser, La Peinture traditionnelle au Canada français, p. 120-21.

32 Morisset, "François Baillairgé (1759-1830) : le peintre ", p. 231.

33 Morisset, "Un primitif, Jean-Baptistc Roy-Audy : son ocuvre ", p. 544.

34 Morisset, La Peinture traditionnelle au Canada français, p. 90-1.

35 Morisset, Novembre 1775, p. 24.

36 Morisset, La Peinture traditionnelle au Canada franf̧ais, p. 26.

37 Morisset, Peintres et tableaux, p. 87-8.

38 Ibid., p. 153.

39 D'autres qualités auraient pu être miscs en valeur, comme la simpliciré ou l'humilité. Toutefois, comme elles ne sont pas systématiquement associées au genre du portrait lui-mêmc, nous avons choisi de les écarter.

40 Lemire, "Gérard Morisset et l'écricure ", p. $2(0)$.

41 Morisset, Peintres et tableaux, p. 118.

42 Morisset, La Peinture traditionnelle au Canada français, p. 137.

13 Morisset, Portraits canadiens du 18 e et 19 e siècles, n.p.

44 Gérard Bouchard, La Pensée impuissante : Echecs el mylhes nationaux canadiens-français (1850-1960), Montrćal, Boréal, 20(1)4, p. 15.

45 Ibid., p. 244.

46. Les dernières remarques s'inspirent de l'introduction théorique de l'ouvrage de Gérard Bouchard (ibid., p. 9-17) cr des discincrions qu'il établit entre la pensée radicale, la pensée organique ct la pensée fragmentaire. Pour les besoins de cc paragraphe, nous nc sommes pas allés jusqu'à approfondir le rôle du mythe dans ces trois types de pensée (bien que cela eût été intércssant). Bouchard ćvitc d'avoir recours au concept d'idéologic pour expliqucr sa rypologie des pensées, probablement parce qu'il ne veut pas réduirc le champ d'application de son système à la sculc idéologie, mais à tous les systèmes de pensée. Dans le cadre de cette analyse ct du nationalisme canadien-français, nous avons jugé que le tcrmc d'idéologie convenait et permettait une meillcure précision.

47 Antoine Prost, Douze lef̧ons sur l'histoire, Paris, Édicions du Seuil, 1996 , p. 87.

48 Ibid., p. 94.

49 Voir plus particulièrement les contributions de Claude Galarneau et de Jacques Robert dans $A$ la découverte du patrimoine avec Ciérard Morisset.

So Cetre manière de dire l'histoire de l'art au Québec n'cst pas ćrcinte. Le catalogue d'exposition sur Louis-Philippe Hébert cst parscmé d'expressions telles que " nos pères ", " notre histoire ", " nos racines ", ... Daniel Drouin, dir., Louis-Philippe Hébert, catalogue d'exposition, Québec, Musée du Québec; Montréal, Musée des beaux-arts de Montréal, 2001, voir par exemplc p. 55, 155, 173-74.
51 Morisset, Coup d'ail sur les arts en Nouvelle-France, p. X.

52 Morisset, La Peinture traditionnelle au Canada français, p. 203-04.

53 Sur ce point, voir Jacques Robcrt, " Gérard Morisset et l'architecture : l'idée et la forme " et André Labergc, "Gérard Morisset : Restaurateur ", dans Á la découverte du patrimoine avec Gérard Morisset.

54 Morisset, La Peinture traditionnelle au Canada français, p. 59.

55 Ibid., p. 127.

56 La société canadienne-française qui voit grandir Gérard Morisset est en plein bourgeonnement intcllcctuel et culturel. Lcs médias (journaux, périodiques, radio, ...) -dont Morisset profitcra pour diffuser son message - sont en effervescence ; l'Écat développc plusieurs mesures pour la formation d'une nouvelle élite canadiennefrançaise et le développement de la culture ; c'est aussi l'ćpoque où l'on voit émerger la figure de l'intellectuel canadien-français. Sur ce point, voir Yvan Lamonde, "Une action intellectuclle", dans Histoire sociale des idées au Québec (1896-1929), Saint-I aurent, Fides, 2004, vol. 2, p. 105-30.

57 Serge Gagnon, Le Québec et ses historiens de 1810 à 1920: La Nouvelle-France de Garneau à Groulx, Qućbec, Presses de l'Univcrsité Laval, 1978, p. 2.

58 Pour en savoir davantage sur les méthodes ct lcs approches que Morisset doit à sa formation françaisc, mais aussi pour lc situcr plus précisément dans l'historiographic canadienne, voir Lauricr Lacroix, "Gérard Morisset et l'histoire de l'art au Qućbcc ", dans À la découverte du patrimoine avec Gérard Morisset.

59) Prost, op. cit., p. 90.

60 Ibid.

61 Denis Monière, Pour comprendre le nationalisme au Québec et ailleurs, Montréal, Presses de l'Université de Montrćal, 2001, p. $95-109$.

62 I.ouis Balthazar, Bilan du nationalisme au Québec, Chicoutimi, J.-M. Tremblay, 2004, 160 p., http://dx.doi.org/doi:10.1522/cla. bal.bil [consulté le 4 décembre 2007]. Réédition ćlectronique de l'édition originale: Montréal, L'Hcxagone, 1986, p. 50 et sq.

6.3 Gérard Bouchard, op. cit., p. 55. Voir aussi (jérard Bouchard, "Lc néonationalisme de Lionel Groulx ", dans Les deux chanoines : contradictions et ambivalence dans la pensée de Lionel (iroulx, Montrćal, Boréal, 2003, p. 35-48.

64 Balthazar, op. cit., p. 21-3.

65 Simon Langlois, dir., "Présentation ", dans Identité et cultures nationales: l'Amérique française en mutation, Québec, Lcs P'resses de l'Université Laval, 1995, p. X.

66 Gérard Bouchard, Genèse des nations et cultures du Nouveau Monde, Montréal, Boréal, 2001, p. 136.

67 Morisset, La Peinture traditionnelle au Canada français, p. 8.

68 Sur le rapport Durham, voir Yvan Lamonde, Histoire sociale des idées au Québec (1760-1896), Saint-Laurent, Fides, 2000, vol. 1, p. 258-68. Pour une analyse du discours historique de FrançoisXavier Garneau, voir Gagnon, op. cit., p. 288-324. 
69 John George I.ambion, 1st earl of Durham, Le rapport Durham, traduit par Denis Bertrand et Albert Desbiens, Montréal, Éditions Sainte-Marie, 1969, p. 121 et sq. ; cité par 1)cnis Monière, Le Développement des idéologies au Québec : des origines à nos jours, Montréal, F́ditions Québcc/Amérique, 1977, p. 150. Le commentaire de ces extraits par Denis Monière témoigne de la dimension émotive qui, plus de cent trente années plus tard, teintc le discours historique vis-à-vis du Rapport Durham.

70 Morisset, Peintres et tableaux, p. IV-V.

71 Ce discours fut prononcé devant plusieurs artistes lors d'un banquet organisé en son honneur. Georges Bellerive, Artistes-peintres canadiens-français : les anciens, 2e édition, Québec, I.ibrairie Beauchemin, 1927, p. 11.

72 H.-R. Casgrain, CFuvres Complètes, 1873, tome I, p. 368 ; cité dans Bouchard, Genèse des nations et cultures du Nouveau Monde, p. 129.

73 Bouchard, Genèse des nations et cultures du Nouveau Monde, p. 111.

74 Ibid., p. 72. Denis Monière fait remarquer que Groulx, comme bien des intellectuels de son temps, utilisait indifféremment les mots : race, peuple, nation et nationalité (Monière, Pour comprendre le nationalisme au Québec et ailleurs, p. 103).

75 L'historiographie récente sur Lionel Groulx s'est peu à peu libérée de l'ancien débat binaire: "Groulx est-il raciste ou non?". C'est ce dont témoignent des ouvrages comme celui Frédéric Boily, La pensée nationaliste de Lionel Groulx, Sillery, Éditions du Scptentrion, 2003, 229 p., ou cncore le collectif dirigé par Robert Boily, dir., Un héritage controversé : nouvelles lectures de Lionel Groulx, Montréal, VLB Éditeur, 2005, 185 p. Pour un regard qui tient scrupuleusement compre des ambiguïtés de ce problème dans l'œuuvre de Groulx, voir Bouchard, Les deux chanoines, principalement aux chapitres IX et X. On retrouvera une réflexion intéressante chez Pierre Hébert, Lionel Groulx et l'Appel de la race, Montrćal, Fides, 1996 , p. 143-45.

76 Jean-Pierre Gaboury, Le Nationalisme de Lionel Groulx : aspects idéologiques, Ottawa, Éditions de l'Université d'Ottawa, 1970 , p. 105.

77 Gustave Le Bon, Lois psychologiques de l'évolution des peuples, Paris, Félix Alcan, 1919, p. 23 ; cicé dans Gaboury, op. cit., p. 105.

78 Sur ce point, voir Bouchard, "Tradition et révisionnisme ", dans Les deux chanoines, p. 49-56.

79 Lionel Groulx (abbé), La Naissance d'une race, 3e ćdition, Montréal, Librairie Granger frères limitée, 1938, p. 65, 67.

80 Ibid., p. 240-41.

81 Lionel Groulx (abbé), Chez nos ancêtres, Montréal, Bibliorhèque de l'Action française, 1920, p. 29.

82 Groulx, La Naissance d'une race, p. 241.

83 Serge Gagnon, Man and his Past : The Nature and Role of Historiography, Montréal, Harvest House, 1982, p. 41.

84 Jacques-Gilles Fournier, "L'idée de tradition artistique dans l'historiographic de l'art canadien : essai d'analyse sociologique ", mémoire de maîtrisc (histoire de l'art), Montréal, Université de
Montréal, 1974, p. 27.

85 Michel Cauchon, Jean-Baptiste Roy-Audy (1788-c.1848), Québec, Ministère des affaires culturelles, 1971, p. 106.

86 Ibid., p. 105.

87 Morisset, Coup d'ceil sur les arts en Nouvelle-France, p. IX-X.

88 Ibid., p. 134-35.

89 Morisset, l'eintres et tableaux, p. VIII.

90 Morisset, Coup d'ceil sur les arts en Nouvelle-France, p. IX.

91 Ibid., p. X.

92 Morisset, La Peinture traditionnelle au Canada français, p. 9-10.

93 Morisset, Coup d'ail sur les arts en Nouvelle-France, p. XI.

94 Bellerive, op. cit., p. 11.

95 Sur ce point, voir la discussion sur l'historicn comme idéologue dans Serge Gagnon, Man and his Past, p. 41-2. 